\title{
Oxidative nanopatterning of titanium generates mesoporous surfaces with antimicrobial properties
}

This article was published in the following Dove Press journal:

International Journal of Nanomedicine

13 May 2014

Number of times this article has been viewed

\author{
Fabio Variola ${ }^{1,2}$ \\ Sylvia Francis Zalzal ${ }^{3}$ \\ Annie Leduc ${ }^{3}$ \\ Jean Barbeau ${ }^{3}$ \\ Antonio $\mathrm{Nanci}^{3}$
}

'Faculty of Engineering, Department of Mechanical Engineering, ${ }^{2}$ Faculty of Science, Department of Physics, University of Ottawa, Ottawa ON, ${ }^{3}$ Faculty of Dental Medicine, Université de Montréal, Montreal, QC, Canada
Correspondence: Antonio Nanci Faculty of Dental Medicine, Université de Montréal, Pavillon Roger-Gaudry, 2900 Edouard Montpetit Boulevard, Montreal, QC, H3T IJ4, Canada

Email antonio.nanci@umontreal.ca

\begin{abstract}
Mesoporous surfaces generated by oxidative nanopatterning have the capacity to selectively regulate cell behavior, but their impact on microorganisms has not yet been explored. The main objective of this study was to test the effects of such surfaces on the adherence of two common bacteria and one yeast strain that are responsible for nosocomial infections in clinical settings and biomedical applications. In addition, because surface characteristics are known to affect bacterial adhesion, we further characterized the physicochemical properties of the mesoporous surfaces. Focused ion beam (FIB) was used to generate ultrathin sections for elemental analysis by energy-dispersive X-ray spectroscopy (EDS), nanobeam electron diffraction (NBED), and high-angle annular dark field (HAADF) scanning transmission electron microscopy (STEM) imaging. The adherence of Staphylococcus aureus, Escherichia coli and Candida albicans onto titanium disks with mesoporous and polished surfaces was compared. Disks with the two surfaces side-by-side were also used for direct visual comparison. Qualitative and quantitative results from this study indicate that bacterial adhesion is significantly hindered by the mesoporous surface. In addition, we provide evidence that it alters structural parameters of $C$. albicans that determine its invasiveness potential, suggesting that microorganisms can sense and respond to the mesoporous surface. Our findings demonstrate the efficiency of a simple chemical oxidative treatment in generating nanotextured surfaces with antimicrobial capacity with potential applications in the implant manufacturing industry and hospital setting.
\end{abstract}

Keywords: mesoporosity, surface characterization, microorganisms, adhesion

\section{Introduction}

In recent years, there has been much interest focused on the influence of nanotopography on cells because this surface feature is in the range of their sensing apparatus. A variety of nanotopographies have now been shown to provide cues that affect gene expression and influence cell destiny and activity. ${ }^{1}$ This remarkable ability to influence cells also extends to bacteria. ${ }^{2}$ It is generally accepted that topographies in the micrometric range promote bacterial adhesion and growth ${ }^{3}$ and that nanoscale features significantly reduce bacterial attachment and proliferation. ${ }^{4-8}$ This, however, does not apply universally, and divergent reports call for additional studies to better understand which, and how, nanofeatures can be exploited to prevent colonization of surfaces by microorganisms..$^{9,10}$

We have been exploiting simple chemical treatments with bicomponent mixtures of acids and oxidants to modify, on the nanoscale, the surface of medically relevant metals. ${ }^{11-13}$ In particular, treatment of titanium with a mixture of $\mathrm{H}_{2} \mathrm{SO}_{4} / \mathrm{H}_{2} \mathrm{O}_{2}$ generates a mesoporous surface that is capable of guiding stem cells and selectively modulating 
cellular events. ${ }^{11,12,14}$ While very promising for regenerative medicine, the ability of such surfaces to control pathogenic microorganisms remains to be evaluated. In this study, we therefore examined the influence of oxidative nanopatterning on common microbial pathogens.

The antimicrobial effects of nanotopography likely result from the synergistic activity of physical and chemical parameters. For example, it is believed that crystallinity of the surface oxide layer of titanium has an impact on bacterial attachment. ${ }^{15}$ We therefore applied high-resolution transmission electron microscopy (TEM) to characterize the thickness, crystallinity, and pore organization of the surface oxide layer resulting from oxidative nanopatterning. This information is fundamental for understanding the interaction of mesoporous surfaces with microorganisms, and eventually, for the ability to rationally design improved surface treatments. We also investigated the adhesion onto the mesoporous surfaces, of two strains of bacteria (ie, Staphylococcus aureus and Escherichia coli) and one yeast (Candida albicans) that are responsible for a large proportion of nosocomial infections in hospitals and biomedical applications. ${ }^{16}$

Our results show that the noncrystalline mesoporous surface layer generated by oxidative nanopatterning hampers the adhesion and/or retention of bacteria. It can also affect the integrity and invasive potential of C. albicans. Together with previous findings, they highlight the potential of simple oxidative chemical treatments for generating medically relevant nanostructured surfaces that compare well with those created by more complex methods.

\section{Materials and methods}

Commercial grade 2 titanium samples (Titanium Industries, Inc., Rockaway, NJ, USA) were treated for 2 hours with a 50:50 mixture of $\mathrm{H}_{2} \mathrm{SO}_{4}(37 \mathrm{~N}) / \mathrm{H}_{2} \mathrm{O}_{2}(30 \%)$ to generate mesoporous surfaces. Consistent with a previous report, ${ }^{13}$ scanning electron microscopy (SEM) (JEOL JSM-7400F; JEOL Ltd, Tokyo, Japan) imaging revealed that surfaces were characterized by a network of nanosized pits, with a diameter of about $20 \mathrm{~nm}$ (Figure 1); these contributed to their greater wettability (as compared with the naturally occurring passive titanium dioxide), ${ }^{17}$ a property that depends on surface charge and chemistry as well as topography. ${ }^{18,19}$ While some surface characterization has previously been carried out on bulk samples, ${ }^{11,13,17}$ we applied high-resolution TEM techniques to examine cross-sectional profiles and thereby achieve more precise physicochemical description of surfaces. Ultrathin cross sections of the treated titanium surface were prepared using a JEOL JIB 4000 focused ion beam (FIB) system. The

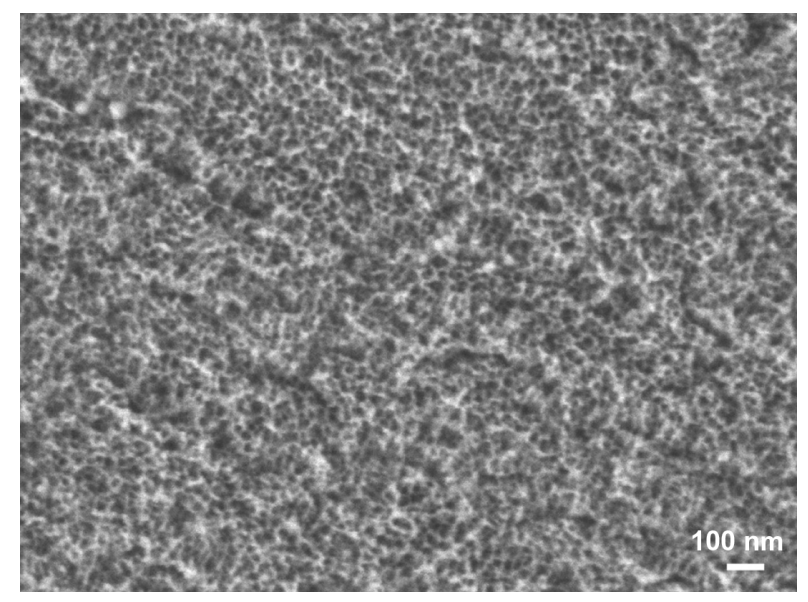

Figure I SEM image of the mesoporous surface generated by oxidative nanopatterning of titanium.

Abbreviation: SEM, scanning electron microscope.

sections were then imaged with a JEOL JEM 2800 TEM outfitted with a high-sensitivity JEOL Centurio wide-angle energy-dispersive X-ray spectroscopy (EDS) detector. Highangle annular dark field (HAADF) scanning transmission electron microscopy (STEM) and nanobeam electron diffraction (NBD) were used to determine the crystallographic nature of the mesoporous surface layer.

SEM was used to investigate bacteria morphology and surface density on smooth and mesoporous surfaces. Experiments were repeated in triplicate, with at least three samples per condition. For E. coli and C. albicans, differences in surface density were evident from images (see "Results"); however, in the case of $S$. aureus, quantitative analysis was necessary to present indisputable evidence of variations in bacteria adherence. In this case, to ensure statistical significance as well as to offset experimental and sampleto-sample variability, bacterial cultures on multiple control and treated samples were carried out in three different sessions, for a total of four samples/condition (smooth versus mesoporous)/session. Images, at 2,000×, were taken in nine randomly selected positions across the sample surface. This approach was consistently applied to the total of 12 samples/ condition, thereby obtaining 108 images/condition. Images were successively processed by analySIS ${ }^{\circledR}$ software (Soft Imaging System GmbH, Münster, Germany) to determine the number of bacteria per micrograph. Bacteria surface density was calculated by first creating four different classes (ie, 0 , $1-25,26-100$, and $>100$ bacteria per image) and then, by determining the percentage of SEM images that fall within each numerical category.

For experiments with E. coli, the untreated half of the disk was covered with a protective dental paraffin layer. After 
treatment in the etching solution, the disk was washed with water, then, paraffin was removed from the protected half with toluene, and lastly, it was rinsed with deionized water, as previously described. ${ }^{14}$

Methicillin-resistant S. aureus (RM J-9160593-2) (kindly provided by Dr Céline Laferrière, Ste-Justine hospital, Montreal, QC, Canada) was prepared in Bacto ${ }^{\mathrm{TM}}$ Tryptic Soy Broth (BD Biosciences). E. coli (ATCC 10798; American Type Culture Collection, Manassas, VA, USA) was prepared in a preparation of Luria-Bertani and Lennox media (Difco ${ }^{\mathrm{TM}}$ LB Broth, Lennox; BD Biosciences), supplemented with glucose $0.2 \%$ (weight/volume [w/v]) (BD Biosciences). C. albicans (ATCC 36802; American Type Culture Collection) was prepared in BBL $^{\text {TM }}$ Trypticase $^{\text {TM }}$ Peptone enzymatic digest of casein (BD Biosciences), 3 g yeast extract (BBL ${ }^{\text {TM}}$; BD Biosciences), $5 \mathrm{~g}$ $\mathrm{NaCl}$ (Thermo Fisher Scientific Inc., Waltham, MA, USA), and $2.5 \mathrm{~g}$ disodium phosphate (Thermo Fisher Scientific Inc.) supplemented with $1.5 \%$ (w/v) glucose. After overnight growth, the cells were pelleted in $50 \mathrm{mM}$ phosphate buffered saline (PBS) at $\mathrm{pH} 7.2$. Bacterial cells $\left(7.5 \times 10^{7} / \mathrm{mL}\right)$ were seeded on disks placed in 24-well multiplates (Costar ${ }^{\circledR}$; Corning Inc., Corning, NY, USA) or in a $60 \times 15 \mathrm{~mm}$ Petri dish. C. albicans was cultured for 48 hours in a media containing $17 \mathrm{~g}$ of Trypticase-Peptone, $3 \mathrm{~g}$ of yeast extract, $5 \mathrm{~g}$ of $\mathrm{NaCl}$, and $2.5 \mathrm{~g}$ of disodium phosphate. A sterile supplement of $1.5 \%(\mathrm{w} / \mathrm{v})$ glucose was added. The $C$. albicans culture was rinsed twice with saline and resuspended in Reasoner's 2A (R2A) broth (BD Biosciences), at a final concentration of $10^{5}$ cells $/ \mathrm{mL}$. A volume of $8.5 \mathrm{~mL}$ of the suspension was placed in a $60 \times 15 \mathrm{~mm}$ Petri dish containing the disks. In all cases, disks were rinsed twice with PBS to eliminate nonadherent cells. Cells were fixed with a solution of $2.5 \%$ glutaraldehyde in phosphate buffer $0.1 \mathrm{M}$, until observed.

\section{Results and discussion}

Contamination of surfaces, fomites, and medical instruments, such as endoscopic and dialysis devices, by opportunistic pathogens is the main culprit for hospital-acquired infections. The costs in human lives, extended hospital stay, and antibiotic therapies add considerable burden on health care. ${ }^{20,21}$ The problem extends to implantable materials, where infection can jeopardize the success of prosthetic devices. For instance, in orthopedics, infection is responsible for the septic failure of almost $5 \%$ of implants, requiring their replacement. ${ }^{7}$ While rigorous cleaning, disinfection, and sterilization protocols will always be necessary, the search is still on for materials that will inherently discourage the spread of microorganisms.
In this study, we applied a simple chemical treatment to create a mesoporous surface layer on titanium. ${ }^{12,13,17}$ We further characterized its structure and atomic order and examined its influence on the adhesion of two bacterial and one yeast strain that are common in medical settings. The resulting mesoporosity had an impact on bacterial adhesion and/or retention as well as on the expression of structural features related to the pathogenic capacity of $C$. albicans.

As previously reported, ${ }^{11-13,17}$ treatment of titanium with a mixture of $\mathrm{H}_{2} \mathrm{SO}_{4} / \mathrm{H}_{2} \mathrm{O}_{2}$ generates a predictable mesoporous titania surface layer comprised of a network of nanopores (Figure 1). Pore structure and diameter (20-25 nm in diameter) and surface roughness (RMS $\sim 17 \mathrm{~nm}$ ) were previously determined by SEM and atomic force microscopy (AFM). ${ }^{13}$ To extend these previous surface analyses, we used FIB to prepare ultrathin sections in cross-sectional profile for TEM characterization of the depth and nature of the mesoporous layer. The network of nanopores extended $40-50 \mathrm{~nm}$ from the surface (Figure 2A), and as revealed by EDS maps of the spatial distribution of oxygen (Figure 2B) and titanium (Figure 2C), it occupied the full thickness of the oxide layer. This finding validates previous results obtained indirectly by coupling Fourier transform infrared (FT-IR) spectroscopy and ellipsometric measurements. ${ }^{22}$ To delineate the metal-oxide interface and determine the atomic order of the surface oxide layer, we applied a combination of TEM approaches. In reliable cross-sectional profiles, nanobeam electron diffraction (NBED) detected a crystalline lattice in the bulk titanium but not in the titania layer (Figure 3 ).

Initial qualitative studies showed less binding of $S$. aureus on the mesoporous surfaces as compared with polished controls, but variability in the difference precluded a definitive interpretation (Figure 4A and B). Quantitative analysis of the binding was therefore carried out in repeat experiments. Figure 4C shows the surface density distribution of $S$. aureus on the treated and control samples. The majority $(71 \%)$ of the SEM images taken on mesoporous surfaces showed fewer than 25 bacteria, and $11 \%$ of them

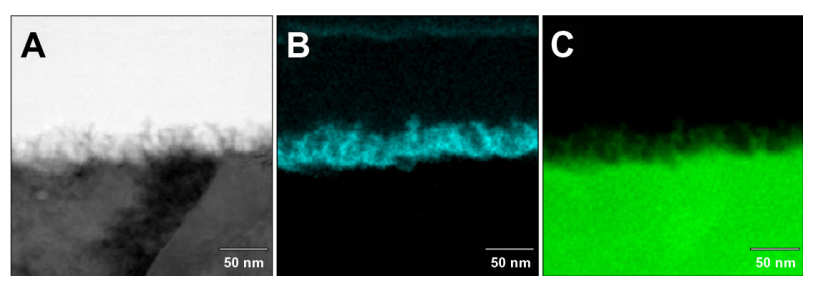

Figure 2 EDS elemental images of oxygen (B) and titanium (C) distribution across the cross section of nanoporous surfaces (A).

Abbreviation: EDS, energy-dispersive X-ray spectroscopy. 


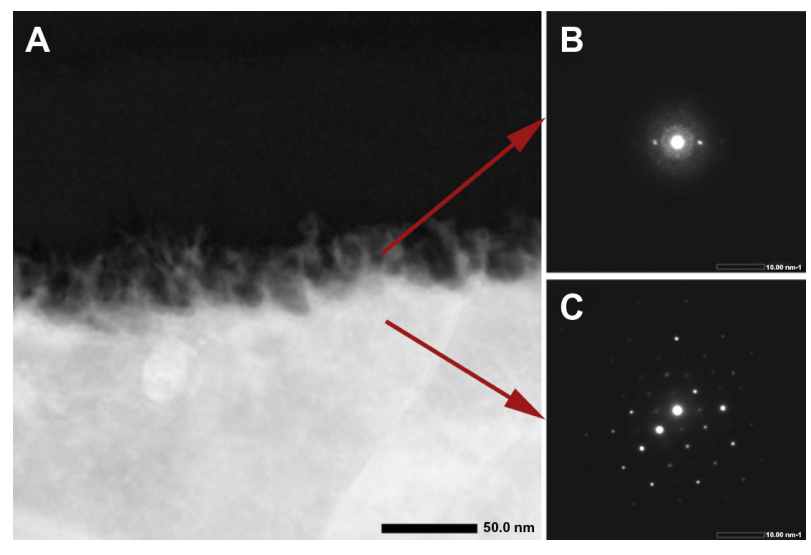

Figure 3 HAADAF (A) and nanodiffraction (B and $\mathbf{C}$ ) analyses were used to examine the metal-oxide interface, revealing the amorphous nature of the surface oxide layer (B) and the typical crystalline lattice of bulk titanium (C).

Abbreviation: HAADAF, high-angle annular dark field.

showed no bacteria at all. Only $5 \%$ of images contained more than 100. For the control samples, the percentage of images with fewer than 25 bacteria was $26 \%$, and $58 \%$ of the regions examined were colonized by more than 100 bacteria. These quantitative results confirm the visual observations and indicate that mesoporous surfaces generated a physicochemical environment that was hostile to binding and/or retention of S. aureus.

The side-by-side bacterial culture approach we used has the advantage of eliminating potential experimental inconsistencies and variations between samples and thus provides direct comparison. SEM imaging of $E$. coli deposited on side-

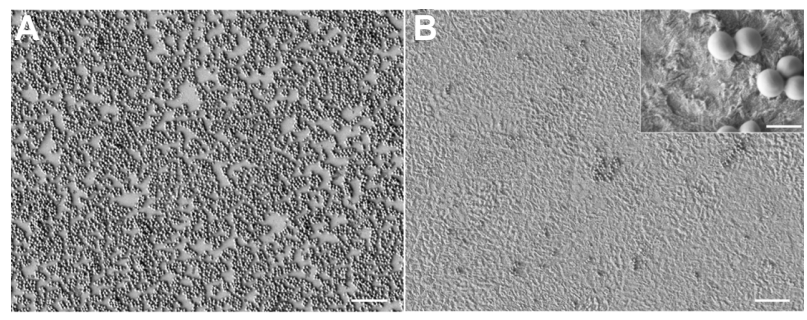

C

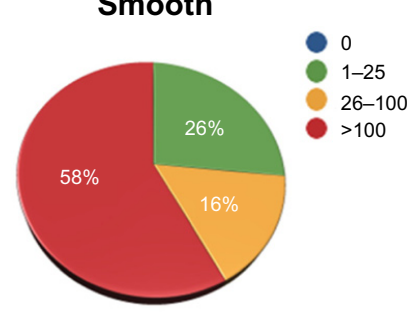

Mesoporous

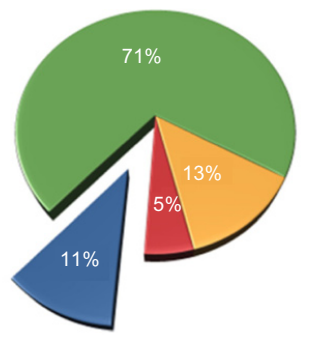

Figure 4 SEM images of Staphylococcus aureus illustrating the adhesion on smooth (A) and mesoporous (B, inset) titanium surfaces. Histogram illustrating the frequency of bacterial density ranges per surface area $(\mathbf{C})$.

Notes: Scale bar $10 \mu \mathrm{m}$ ( $1 \mu \mathrm{m}$ inset). Image analysis and bacteria counting were carried out for a total of 12 samples/condition (ie, 108 images/condition).

Abbreviation: SEM, scanning electron microscope. by-side smooth/mesoporous surfaces displayed a visually and readily evident reduction in adhesion on the treated half of the titanium disks that did not require further quantitative evaluation for validation (Figure 5). A comparative study of bacterial binding and retention revealed the presence of type 1 fimbriae on bacteria cultured on smooth gold, while those on nanoporous gold did not develop this adhesive organelle. ${ }^{23}$ The absence of this organelle suggests weaker adhesion onto the nanostructured surface and correlated with activation of cpxP (periplasmic adaptor protein) and $\operatorname{deg} P$ (serine endoprotease) genes implicated in the bacterial envelope stress response and upregulation of fimbrial recombinase FimE. ${ }^{23}$ Clearly, nanotopography can change the "normal" behavior of bacteria.

Quantitative experiments with C. albicans were more complex to interpret because of the filamentous nature of this microorganism. C. albicans distinctively produces hyphae when it germinates. These branching filamentous structures are implicated in its capacity to invade tissues. ${ }^{24}$ SEM overall showed fewer long filaments on the mesoporous surface (Figure 6). In addition, the overall structural integrity of the microorganism appeared affected, exhibiting a crenated morphology compared with controls. Although qualitative, these results suggest that the physicochemical environment of the mesoporous surface created by the oxidative treatment may affect the integrity and invasive potential of C. albicans. Taken together, data from bacteria and yeast studies suggest that microorganisms possess a fine sensing capacity that can respond to nanoscale features and changes in the overall physicochemical environment.

The mechanisms by which the surface mesoporosity created by oxidative treatment with $\mathrm{H}_{2} \mathrm{SO}_{4} / \mathrm{H}_{2} \mathrm{O}_{2}$ achieves

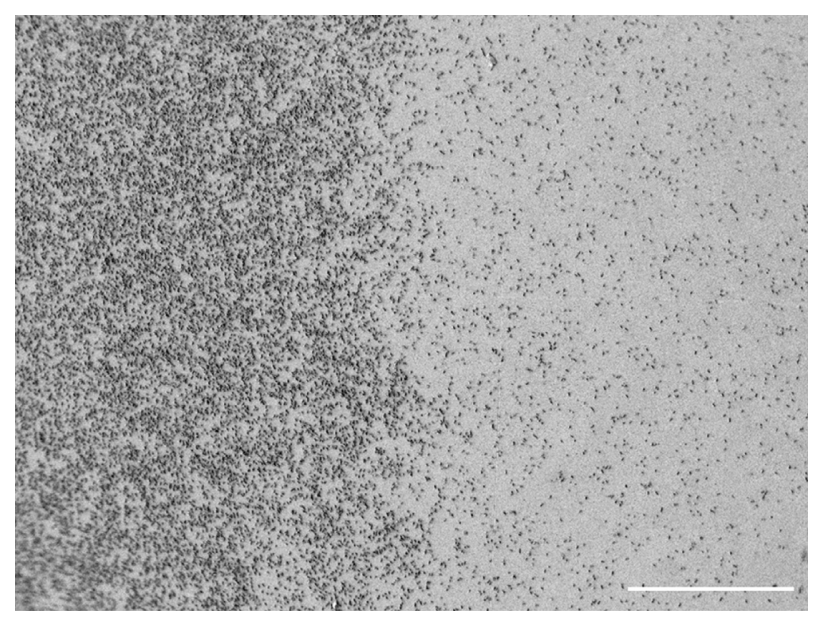

Figure 5 SEM image of Escherichia coli on side-by-side smooth (left)/mesoporous (right) surface.

Note: Scale bar $100 \mu \mathrm{m}$.

Abbreviation: SEM, scanning electron microscope. 

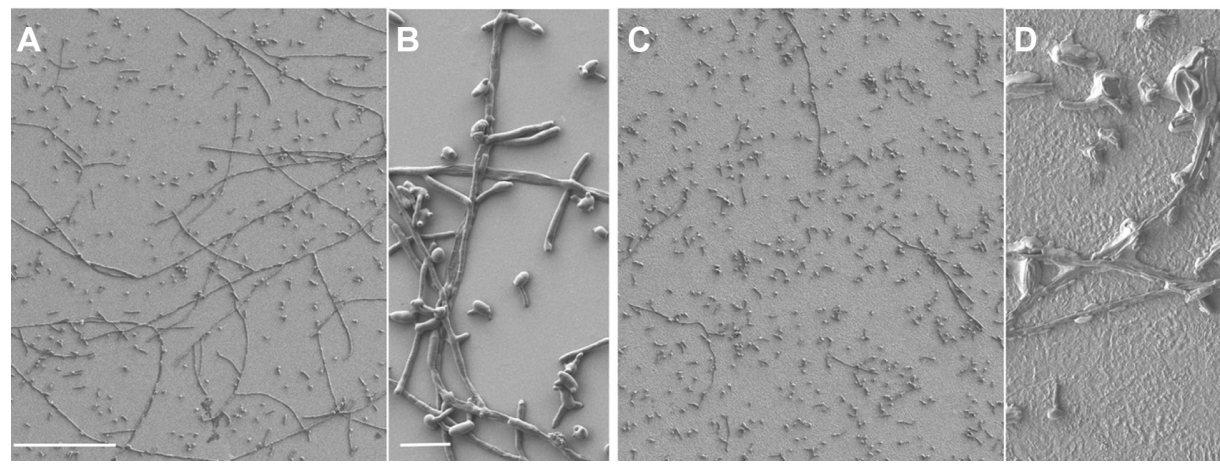

Figure 6 SEM images of Candida albicans adhesion on smooth (A and B) and mesoporous (C and $\mathbf{D})$ titanium surfaces. Note the paucity of hyphae (C) and altered crenated appearance of the yeast cells (D) on the treated surface.

Notes: Scale bar $100 \mu \mathrm{m}$ (A and $\mathbf{C}), 10 \mu \mathrm{m}$ (B and $\mathbf{D})$.

Abbreviation: SEM, scanning electron microscope.

antimicrobial effects remain to be determined. Although the anatase crystalline form of titanium oxide was shown to significantly reduce bacterial adhesion, ${ }^{15,25}$ it does not appear to have been a predominant factor in this study, since our mesoporous layer was amorphous and likewise, exhibited antimicrobial activity. It should be considered that the anodization methods used to create crystalline anatase may also generate nanostructures with antibacterial properties. ${ }^{6,7}$ Mesoporous amorphous oxide can be converted into a crystalline layer by thermal annealing, while preserving nanotopography. ${ }^{26}$ It will thus be interesting to determine whether the described antimicrobial capacity could be enhanced by rendering the mesoporous surface crystalline.

The effects of potential chemical residues on the observed antimicrobial properties can be excluded from the contributing factors by previous X-ray photoelectron spectroscopy (XPS) analysis, which showed no significant acid remnants on the mesoporous surfaces. ${ }^{13}$ This was also confirmed by the EDS analysis carried out in this study. In addition, a recent report has concluded that traditional surface roughness parameters are not the only determinants of bacterial adhesion and that the spatial distribution of nanofeatures may also have predictive value. ${ }^{27}$ Especially when considering some conflicting reports in the literature, the rational exploitation of nanotopography for controlling bacterial colonization will require the evaluation of additional nanostructured surfaces and a more stringent consideration of the synergistic influence of the multiple physicochemical parameters that characterize surfaces.

There are circumstances where microtextured surfaces (eg, sandblasted or beaded implants) are medically advantageous (eg, to favor implant-bone mechanical interlocking), but these may favor bacterial adhesion. ${ }^{3}$ The superimposition of nanotexture on microtextured surfaces could represent a promising strategy to discourage bacterial adhesion on microtopographical surfaces that provide good biomechanical retention. Here it should be pointed out that oxidative nanopatterning is a flexible approach that can directly produce microtopography, with superimposed mesoporosity. ${ }^{1}$

\section{Conclusion}

Since adhesion is the initial step in the bacterial colonization of surfaces, ${ }^{28}$ the capacity to interfere with this activity holds the key to devising antibacterial surfaces capable of preventing the subsequent cascade of biofilm formation. We have demonstrated that oxidative nanopatterning can be applied to titanium to reduce adhesion and/or retention of $S$. aureus and E. coli, and influence the aggregation capacity and integrity of $C$. albicans, all three microorganisms responsible for implant-related and hospital-acquired infections. Reduction of bacterial adhesion may possibly involve interference with the production/action of bacterial adhesins ${ }^{29}$ or the formation attachment structures, such as fimbriae. ${ }^{23}$ Oxidative nanopatterning offers the advantage of being applicable to a variety of medically relevant bulk metals, similarly to electrochemical methods currently used to engender nanoporosities (eg, anodization).${ }^{11}$ The approach can also be readily applied to sputtered titanium thin films $\mathrm{s}^{30}$ applied as coatings on nonmetallic substrates and is especially pertinent to the complex geometries of prosthetic devices used in cardiology, dentistry, and orthopedics. ${ }^{1}$ Just like titania nanotubular surfaces, ${ }^{31-35}$ the presence of nanoconfined volumes within the mesoporosity could be exploited to store bioactive agents and antibiotics, and modulate their release over time. ${ }^{36}$ Taken together, these characteristics make oxidative nanopatterning a valuable strategy to impart antibacterial properties to metal surfaces found in industry and hospitals, and to prosthetic devices. Because the created nanoporous surface has shown 
beneficial cellular and tissue effects, ${ }^{11,12}$ and as shown here, can inherently control bacterial adhesion, it is particularly promising for medical implants, where the tissue and immune response ultimately determine clinical success.

\section{Acknowledgments}

FV acknowledges start-up funding from the Faculty of Engineering and University of Ottawa and financial support from the Natural Sciences and Engineering Research Council of Canada (NSERC). AN acknowledges funding from the Canada Foundation for Innovation, Canadian Institutes of Health Research (CIHR) and NSERC. We thank Dr Ji-Hyun Yi for the preparation of titanium disks with dual surfaces, and JEOL Japan for assistance with FIB and TEM analysis.

\section{Disclosure}

The authors report no conflicts of interest in this work.

\section{References}

1. Variola F, Brunski JB, Orsini G, Tambasco de Oliveira P, Wazen R, Nanci A. Nanoscale surface modifications of medically relevant metals: state-of-the art and perspectives. Nanoscale. 2011;3(2): 335-353.

2. Rodrigues LR. Inhibition of bacterial adhesion on medical devices. Adv Exp Med Biol. 2011;715:351-367.

3. Wu Y, Zitelli JP, TenHuisen KS, Yu X, Libera MR. Differential response of Staphylococci and osteoblasts to varying titanium surface roughness. Biomaterials. 2011;32(4):951-960.

4. Puckett SD, Taylor E, Raimondo T, Webster TJ. The relationship between the nanostructure of titanium surfaces and bacterial attachment. Biomaterials. 2010;31(4):706-713.

5. Truong VK, Lapovok R, Estrin YS, et al. The influence of nano-scale surface roughness on bacterial adhesion to ultrafine-grained titanium. Biomaterials. 2010;31(13):3674-3683.

6. Ercan B, Kummer KM, Tarquinio KM, Webster TJ. Decreased Staphylococcus aureus biofilm growth on anodized nanotubular titanium and the effect of electrical stimulation. Acta Biomater. 2011;7(7): 3003-3012.

7. Ercan B, Taylor E, Alpaslan E, Webster TJ. Diameter of titanium nanotubes influences anti-bacterial efficacy. Nanotechnology. 2011;22(29):295102.

8. Mitik-Dineva N, Wang J, Mocanasu RC, Stoddart PR, Crawford RJ, Ivanova EP. Impact of nano-topography on bacterial attachment. Biotechnol J. 2008;3(4):536-544.

9. Rizzello L, Cingolani R, Pompa PP. Nanotechnology tools for antibacterial materials. Nanomedicine (Lond). 2013;8(5): $807-821$.

10. Hasan J, Crawford RJ, Ivanova EP. Antibacterial surfaces: the quest for a new generation of biomaterials. Trends Biotechnol. 2013;31(5): 295-304.

11. Vetrone F, Variola F, Tambasco de Oliveira P, et al. Nanoscale oxidative patterning of metallic surfaces to modulate cell activity and fate. Nano Lett. 2009;9(2):659-665.

12. Variola F, Yi JH, Richert L, Wuest JD, Rosei F, Nanci A. Tailoring the surface properties of Ti6Al4V by controlled chemical oxidation. Biomaterials. 2008;29(10):1285-1298.
13. Yi JH, Bernard C, Variola F, et al. Characterization of a bioactive nanotextured surface created by controlled chemical oxidation of titanium. Surf Sci. 2006;600(19):4613-4621.

14. Richert L, Vetrone F, Yi JH, et al. Surface nanopatterning to control cell growth. Adv Mater. 2008;20(8):1488-1492.

15. Del Curto B, Brunella MF, Giordano C, et al. Decreased bacterial adhesion to surface-treated titanium. Int J Artif Organs. 2005;28(7): 718-730.

16. Calfee DP. Crisis in hospital-acquired, healthcare-associated infections. Annu Rev Med. 2012;63:359-371.

17. Variola F, Lauria A, Nanci A, Rosei F. Influence of treatment conditions on the chemical oxidative activity of $\mathrm{H} 2 \mathrm{SO} 4 / \mathrm{H} 2 \mathrm{O} 2$ mixtures for modulating the topography of titanium. Adv Eng Mater. 2010;11(12):B227-B234.

18. Puah LS, Sedev R, Fornasiero D, Ralston J, Blake T. Influence of surface charge on wetting kinetics. Langmuir. 2010;26(22):17218-17224.

19. Meiron TS, Marmur A, Saguy IS. Contact angle measurement on rough surfaces. J Colloid Interface Sci. 2004;274(2):637-644.

20. Falagas ME, Roussos N, Vardakas KZ. Relative frequency of albicans and the various non-albicans Candida spp among candidemia isolates from inpatients in various parts of the world: a systematic review. Int J Infect Dis. 2010;14(11):e954-e966.

21. Manning SD. Escherichia Coli Infections (Deadly Diseases and Epidemics). New York: Chelsea House Publications; 2004

22. Variola F, Nanci A, Rosei F. Assessment of the titanium dioxide absorption coefficient by grazing-angle Fourier transform infrared and ellipsometric measurements. Appl Spectrosc. 2009;63(10):1187-1190.

23. Rizzello L, Galeone A, Vecchio G, Brunetti V, Sabella S, Pompa PP. Molecular response of Escherichia coli adhering onto nanoscale topography. Nanoscale Res Lett. 2012;7(1):575.

24. Sudbery PE. Growth of Candida albicans hyphae. Nat Rev Microbiol. 2011;9(10):737-748.

25. Giordano C, Saino E, Rimondini L, et al. Electrochemically induced anatase inhibits bacterial colonization on Titanium Grade 2 and Ti6Al4V alloy for dental and orthopedic devices. Colloids Surf B Biointerfaces. 2011;88(2):648-655.

26. Variola F. Surface Modifications of Biocompatible Metals to Improve Biological Response [PhD thesis]. Varennes: Institut National de la Recherche Scientifique - Énergie, Matériaux et Télècommunications; 2010.

27. Webb HK, Boshkovikj V, Fluke CJ, et al. Bacterial attachment on subnanometrically smooth titanium substrata. Biofouling. 2013;29(2): $163-170$.

28. Temenoff JS, Mikos AG. Biomaterials: the Intersection of Biology and Materials Science. Upper Saddle River, NJ: Prentice Hall; 2008.

29. Ofek I, Hasty DL, Sharon N. Anti-adhesion therapy of bacterial diseases: prospects and problems. FEMS Immunol Med Microbiol. 2003;38(3): 181-191.

30. Richert L, Variola F, Rosei F, Wuest JD, Nanci A. Adsorption of proteins on nanoporous Ti surfaces. Surf Sci. 2010;604(17-18):1445-1451.

31. Brammer KS, Frandsen CJ, Jin S. TiO2 nanotubes for bone regeneration. Trends Biotechnol. 2012;30(6):315-322.

32. Yao C, Webster TJ. Prolonged antibiotic delivery from anodized nanotubular titanium using a co-precipitation drug loading method. J Biomed Mater Res B Appl Biomater. 2009;91(2):587-595.

33. Roy P, Berger S, Schmuki P. TiO2 nanotubes: synthesis and applications. Angew Chem Int Ed Engl. 2011;50(13):2904-2939.

34. Popat KC, Eltgroth M, LaTempa TJ, Grimes CA, Desai TA. Titania nanotubes: a novel platform for drug-eluting coatings for medical implants? Small. 2007;3(11):1878-1881.

35. Peng L, Mendelsohn AD, LaTempa TJ, Yoriya S, Grimes CA, Desai TA. Long-term small molecule and protein elution from $\mathrm{TiO} 2$ nanotubes. Nano Lett. 2009;9(5):1932-1936.

36. Ketabchi A, Komm K, Miles-Rossouw M, Cassani DAD, Variola F. Nanoporous titanium surfaces for sustained elution of proteins and antibiotics. PLoS ONE. 2014;9(3): e92080. doi:10.1371/journal. pone.0092080. 
International Journal of Nanomedicine

Dovepress

\section{Publish your work in this journal}

The International Journal of Nanomedicine is an international, peerreviewed journal focusing on the application of nanotechnology in diagnostics, therapeutics, and drug delivery systems throughou the biomedical field. This journal is indexed on PubMed Central, MedLine, CAS, SciSearch $®$, Current Contents ${ } /$ Clinical Medicine,
Journal Citation Reports/Science Edition, EMBase, Scopus and the Elsevier Bibliographic databases. The manuscript management system is completely online and includes a very quick and fair peer-review system, which is all easy to use. Visit http://www.dovepress.com/ testimonials.php to read real quotes from published authors.

Submit your manuscript here: http://www.dovepress.com/international-journal-of-nanomedicine-journal 